\title{
M-Center in Neutron Irradiated 4H-SiC
}

\author{
Ivana Capan ${ }^{1, *}$, Tomislav Brodar ${ }^{1}$, Takahiro Makino ${ }^{2}$, Vladimir Radulovic ${ }^{3}$ and Luka Snoj ${ }^{3}$
}

1 Ruđer Bošković Institute, Bijenička cesta 54, 10000 Zagreb, Croatia; capan@irb.hr (I.C.); TomislavBrodar@gmail.com (T.B.)

2 National Institutes for Quantum and Radiological Science and Technology, 1233 Watanuki, Takasaki Gunma 370-1292, Japan; makino.takahiro@qst.go.jp (T.M.)

3 Jožef Stefan Institute, Jamova cesta 39, 1000 Ljubljana, Slovenia; vladimir.radulovic@ijs.si (V.R.); luka.snoj@ijs.si (L.S.)

* Correspondence: capan@irb.hr;

\begin{abstract}
We report on metastable defects introduced in n-type 4H-SiC material by epithermal and fast neutron irradiation. The epithermal and fast neutron irradiation defects in $4 \mathrm{H}-\mathrm{SiC}$ are much less explored compared to electron or proton irradiation induced defects. In addition to silicon vacancy (Vsi) and carbon antisite-carbon vacancy (CAV) complex, the neutron irradiation has introduced four deep level defects, all arising from the metastable defect, the M-center. The metastable deep level defects were investigated by deep level transient spectroscopy (DLTS), high-resolution Laplace DLTS (L-DLTS) and isothermal DLTS. The existence of the fourth deep level $\mathrm{M}_{4}$, recently observed in ion implanted $4 \mathrm{H}-\mathrm{SiC}$, has been additionally confirmed in neutron irradiated samples. The isothermal DLTS technique has been proven as a useful tool for studying the metastable defects.
\end{abstract}

Keywords: defects; 4H-SiC; DLTS; neutrons;

\section{Introduction}

The metastable defects whose configuration depends on bias and/or thermal treatments, and whose behavior was not always easy or fully understood, have a special place in studying the deep level defects in semiconductors. One of those bi-stable defects is the $\mathrm{M}$-center in $4 \mathrm{H}-\mathrm{SiC}$ material, reported for the first time almost two decades ago [1]. Since then, M-center has been observed upon the low energy electron irradiation [2,3], $2.5 \mathrm{MeV}$ proton irradiation [1,4-6] and $2 \mathrm{MeV}$ He ion implantation [7] of n-type $4 \mathrm{H}-\mathrm{SiC}$. Comprehensive experimental studies and analysis have been performed using mostly the deep level transient spectroscopy (DLTS).

The M-center in n-type $4 \mathrm{H}-\mathrm{SiC}$ is a bi-stable defect whose configuration depends on bias and thermal treatments imposed to the samples. Therefore, by adjusting the bias and following well-defined annealing procedures [7], M-center can be placed in a selected configuration before DLTS measurement. It has been widely accepted among the research community to refer to the M-center configurations as A and B.

Since the first published reports of the M-center, three deep level defects were assigned to it. The $\mathrm{M}_{1}(0.41-0.43 \mathrm{eV})$ and $\mathrm{M}_{3}(0.75-0.9 \mathrm{eV})$ deep levels were observed in configuration $\mathrm{A}$, while $\mathrm{M}_{2}(0.63-0.70 \mathrm{eV})$ deep level was observed in configuration $\mathrm{B}$ [16]. In addition to these three deep levels arising from the M-center, Nielsen et al. [6] have proposed the existence of a fourth deep level in configuration B and labeled it as $\mathrm{M}_{4}$. However, due to the technical limitations of DLTS i.e., the fact that the technique itself involves a temperature scan, the $\mathrm{M}_{4}$ deep level was not accessible for many years.

Recently, Coutinho et al. [8] have provided the direct evidence on the existence of the $\mathrm{M}_{4}(0.86 \mathrm{eV})$ deep level in $4 \mathrm{H}-\mathrm{SiC}$. Combining the advanced first-principles calculations and isothermal DLTS, it was demonstrated that the properties of M-center, including bistability, annealing, reconfiguration kinetics, and electronic levels match those of the carbon self-interstitial. 
In this work, we have applied DLTS, high-resolution Laplace DLTS (L-DLTS) and isothermal DLTS to study the metastable defects in $4 \mathrm{H}-\mathrm{SiC}$ material introduced by epithermal and fast neutron irradiation. The main aim of this work is to investigate the much less studied neutron irradiation induced defects primarily due to the growing interest and applications of $4 \mathrm{H}-\mathrm{SiC}$ materials for neutron detection [9]. In addition, our intention is to compare these findings with the recently published study on ion implantation induced defects and to further explore the possibilities of isothermal DLTS technique.

\section{Materials and Methods}

For this study, we have used n-type nitrogen-doped $4 \mathrm{H}-\mathrm{SiC}$ epitaxial layers $25 \mu \mathrm{m}$ thick. The epilayers were grown on an $8^{\circ}$ off-cut silicon face of a $350 \mu \mathrm{m}$ thick $4 \mathrm{H}-\mathrm{SiC}$ (0001) wafer without a buffer layer. The Schottky barriers diodes (SBDs) were formed by thermal evaporation of nickel through a metal mask with a patterned square aperture of $1 \mathrm{~mm} \times 1 \mathrm{~mm}$, while Ohmic contacts were formed on the backside of the silicon carbide substrate by nickel sintering at $950{ }^{\circ} \mathrm{C}$ in an Ar atmosphere.

The SBDs were irradiated with epithermal and fast neutrons at the Jozef Stefan Institute (JSI) TRIGA reactor in Ljubljana, Slovenia. The selected neutron fluence was $10^{13} \mathrm{~cm}^{-2}$. Thermal neutrons with energy less than $0.55 \mathrm{eV}$ were filtered by irradiating the Schottky barrier diodes inside a cadmium box with a wall thickness of $1 \mathrm{~mm}$. The neutron energy spectrum and irradiation settings are given elsewhere [10].

The temperature dependent current-voltage (I-V-T) and capacitance-voltage $(\mathrm{C}-\mathrm{V}-\mathrm{T})$ measurements are performed by Keithley 4200 SCS (Keithley Instruments, Cleveland, USA). DLTS, L-DLTS and isothermal DLTS measurements were performed using Boonton 7200 capacitance meter (Boonton Electronics, New Jersey, USA) and NI PCI-6521 data acquisition device (NI, Austin, USA). Reverse bias and electrical filling pulses were applied to the SBDs using the analog output of the acquisition device. The stable temperature control of the SBD in OptistatDN cryostat (Oxford Instruments, Oxfordshire, England) was realized using Lakeshore 332 and Lakeshore 335 temperature controllers (Lake Shore Cryotronics, Inc., Ohio, USA). The sample temperature was constant during isothermal DLTS measurements, changing less than $0.05 \mathrm{~K}$. Isothermal DLTS technique is a very useful technique which enables studies of the deep level transformation kinetics at the same temperature [11].

Three different annealing procedures were used to control the configuration of the $\mathrm{M}$-center prior to measurements. Detailed descriptions on annealing procedures are given elsewhere [7].

\section{Results and discussion}

Figure 1 shows the free-carrier concentration profiles of as-grown, $2 \mathrm{MeV}$ He ion implanted, and epithermal and fast neutron irradiated n-type $4 \mathrm{H}-\mathrm{SiC}$ samples (solid lines, left y axis) and the calculated vacancy concentration profiles (dashed lines, right y axis). The displacement damage profiles i.e., the vacancy concentration profiles are simulated using the SRIM code [12] and FLUKA software [13] for the ion implanted and neutron irradiated $4 \mathrm{H}-\mathrm{SiC}$, respectively.

As expected, the free carrier concentration profiles are significantly different in ion implanted and neutron irradiated samples. The reason is that epithermal and fast neutron irradiation introduces damage homogenously through the volume, while the ion implantation introduces damage with a well-defined depth profile (Figure 1). To avoid region of $2 \mathrm{MeV} H e$ ion projected range at $4.8 \mu \mathrm{m}$, reverse voltage of $-4 \mathrm{~V}$ corresponding to a depletion width of $<4 \mu \mathrm{m}$ was selected for DLTS measurements [7]. Such restrictions are redundant in the case of neutron irradiated samples, and reverse voltage up to $-10 \mathrm{~V}$ was used. 


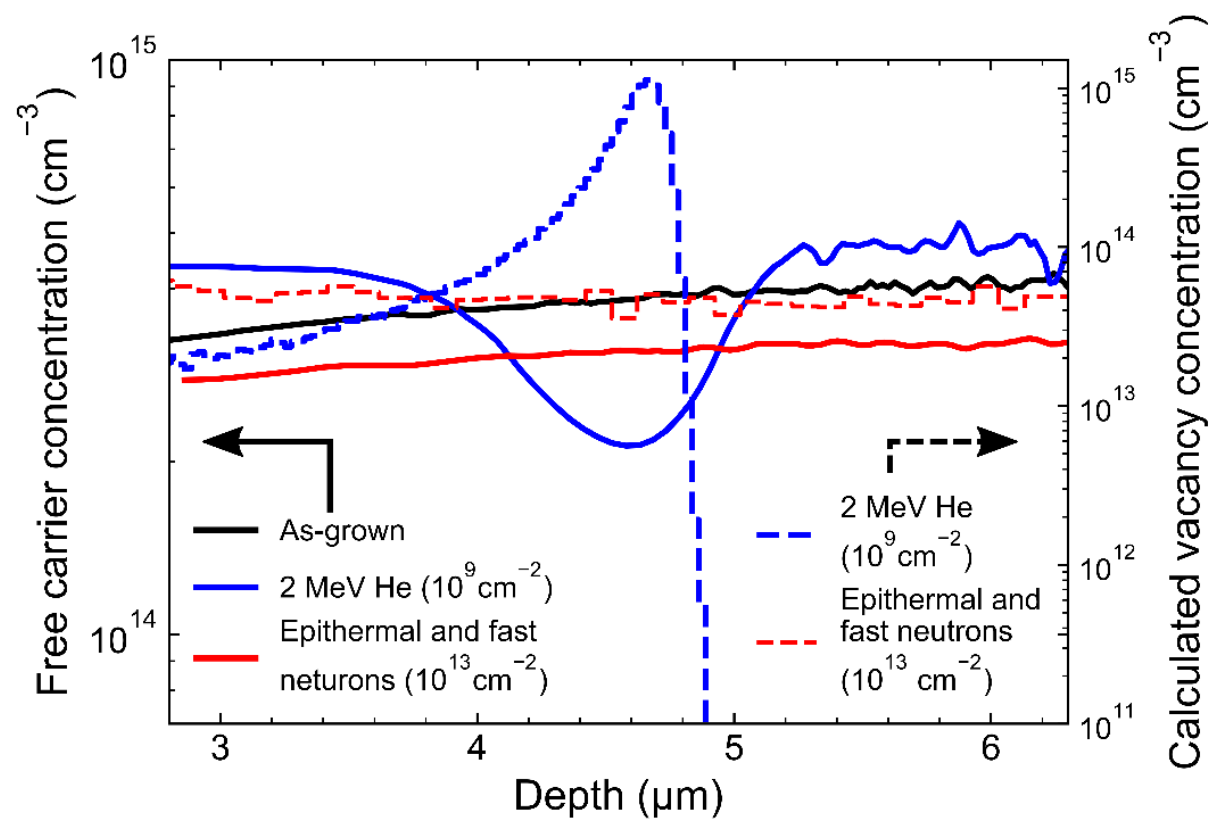

Figure 1. Free-carrier concentration profiles of as-grown, ion implanted and neutron irradiated n-type $4 \mathrm{H}-\mathrm{SiC}$ samples (solid lines, left y axis) and the calculated vacancy concentration profiles (dashed lines, right y axis). The free carrier concentration profiles are obtained from the $\mathrm{C}-\mathrm{V}$ measurements.

The reasons why we have selected epithermal and fast neutrons to introduce deep level defects in $4 \mathrm{H}-\mathrm{SiC}$ material are multiple. The growing interest in neutron radiation detection based on $4 \mathrm{H}-\mathrm{SiC}$ material being just one of them [9]. Additional reasons are i) the homogenously distributed damage through the volume (as clearly shown in Figure 1), which is rather important for techniques such as DLTS (that scans over volume), and ii) the fact that epithermal and fast neutron irradiation introduces the same electrically active defects as ion implantation into $4 \mathrm{H}-\mathrm{SiC}$ [10]. Those defects are known as $\mathrm{S}_{1}(0.40$ $\mathrm{eV})$ and $\mathrm{S}_{2}(0.70 \mathrm{eV})$, and already assigned to $\mathrm{V}_{\mathrm{si}}(-3 /=)$ and $\mathrm{V}_{\mathrm{si}}(=/-)$ charge state transitions of the silicon vacancy [14], respectively.

Figure 2 shows DLTS spectra for the neutron irradiated sample in two configurations, $\mathrm{A}$ and $\mathrm{B}$. The M-center was transformed to configuration B or configuration A by annealing at $450 \mathrm{~K}$ (for 20 minutes) and cooling down without applying bias $(0 \mathrm{~V})$ or by $20 \mathrm{~min}$ annealing at the temperature of $340 \mathrm{~K}$ and cooling down with the applied bias $(-30 \mathrm{~V})$, respectively. 


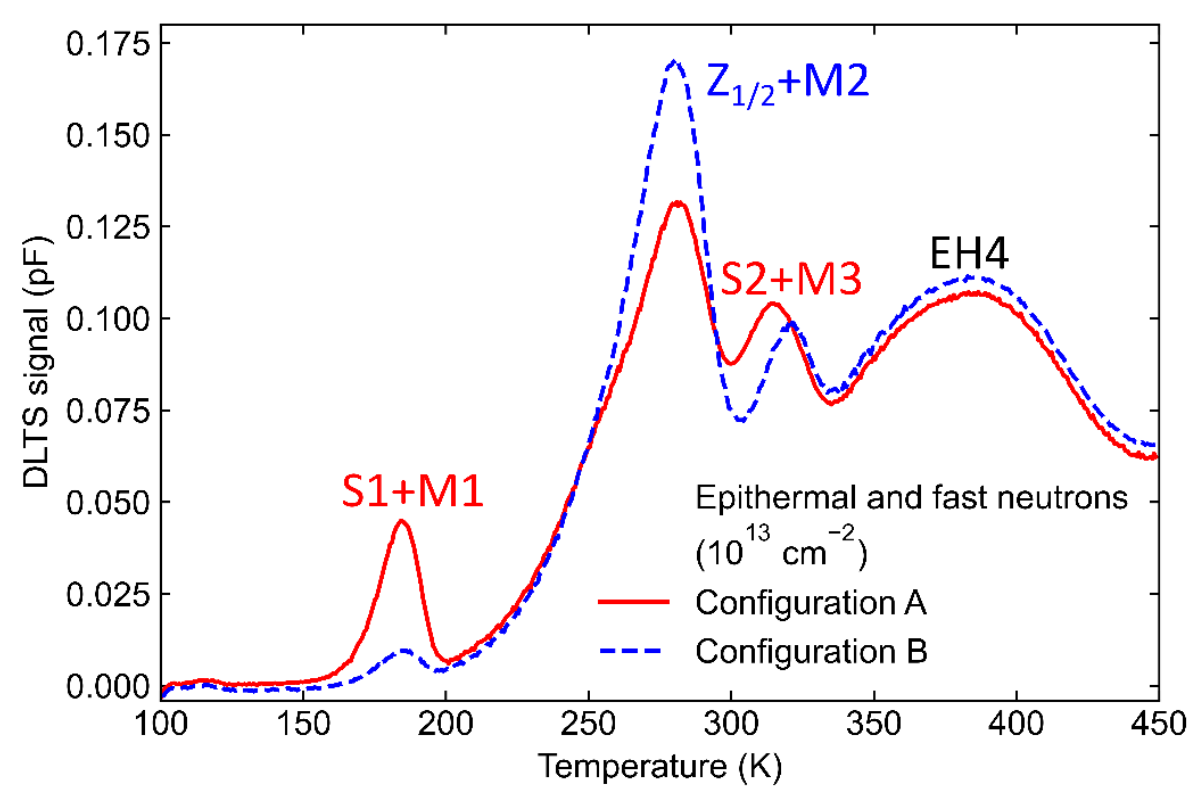

Figure 2. DLTS spectra of neutron irradiated $4 \mathrm{H}-\mathrm{SiC}$ sample in two configurations of the Mcenter, configuration A (red, solid) and configuration B (blue, dashed).

In addition to a well-known $\mathrm{Z}_{1 / 2}$ deep level defect assigned to a transition between double negative and neutral charge state of carbon vacancy $V_{C}(=/ 0)$ [15], three deep levels with activation energies of $0.4,0.7$, and $0.9 \mathrm{eV}$ below the conduction band minimum are detected. Two deep levels at 0.4 and $0.7 \mathrm{eV}$ are assigned to already mentioned $\mathrm{S}_{1}$ and $\mathrm{S}_{2}$, while $0.9 \mathrm{eV}$ deep level labeled as $\mathrm{EH}_{4}$ is assigned to the carbon antisite-carbon vacancy (CAV) complex [16].

As seen in Figure 2., the $\mathrm{M}_{1}$ and $\mathrm{M}_{3}$ deep levels observed in configuration A overlap with $S_{1}$ and $S_{2}$ deep levels, while the $M_{2}$ deep level observed in configuration B overlaps with $Z_{1 / 2}$. This result is consistent with previously published DLTS results on $2 \mathrm{MeV} H \mathrm{He}$ ion implantation induced defects [7].

To obtain more information about the metastable defects in neutron irradiated samples, we have applied L-DLTS to investigate $S_{1}$ and $M_{1}$ deep level defects. Figure 3 shows L-DLTS spectra for neutron irradiated sample measured in configurations A and B. While keeping the sample in configuration B (only $S_{1}$ deep level is detected), two emission lines arising from $S_{1}$ are observed. The $S 1$ Laplace emission line splitting has already been reported [14]. The situation has slightly changed as we have set the sample in configuration A. As expected, (see Figure 2), the signal due to the $\mathrm{M}_{1}$ deep level is rising, and it overlaps with one of the $S_{1}$ Laplace emission lines (Figure 3). To the best of our knowledge, this is a first-time metastable defect in $4 \mathrm{H}-\mathrm{SiC}$ has been observed by L-DLTS.

According to the modelling studies [8], the $\mathrm{M}_{1}$ deep level is assigned to the carbon self-interstitial (Ci) residing on the hexagonal lattice site $(-h)$. If the $S_{1}$ emission lines splitting ( $\mathrm{S}_{1}$ and $\mathrm{S} 1_{2}$ in Figure 3 ) corresponds to silicon vacancies $\left(\mathrm{V}_{\mathrm{Si}}\right)$ residing on two different lattice sites, like the carbon vacancy $\left(V_{c}\right)[16]$, then we can assign two observed L-DLTS emission lines $S 1_{1}$ and $S 1_{2}$ to $\mathrm{V}_{\mathrm{Si}}$ residing on two lattices sites, cubic $(-k)$ and hexagonal ($h)$, respectively, while the $\mathrm{M}_{1}$ deep level resides on hexagonal $(-h)$ lattice site. 


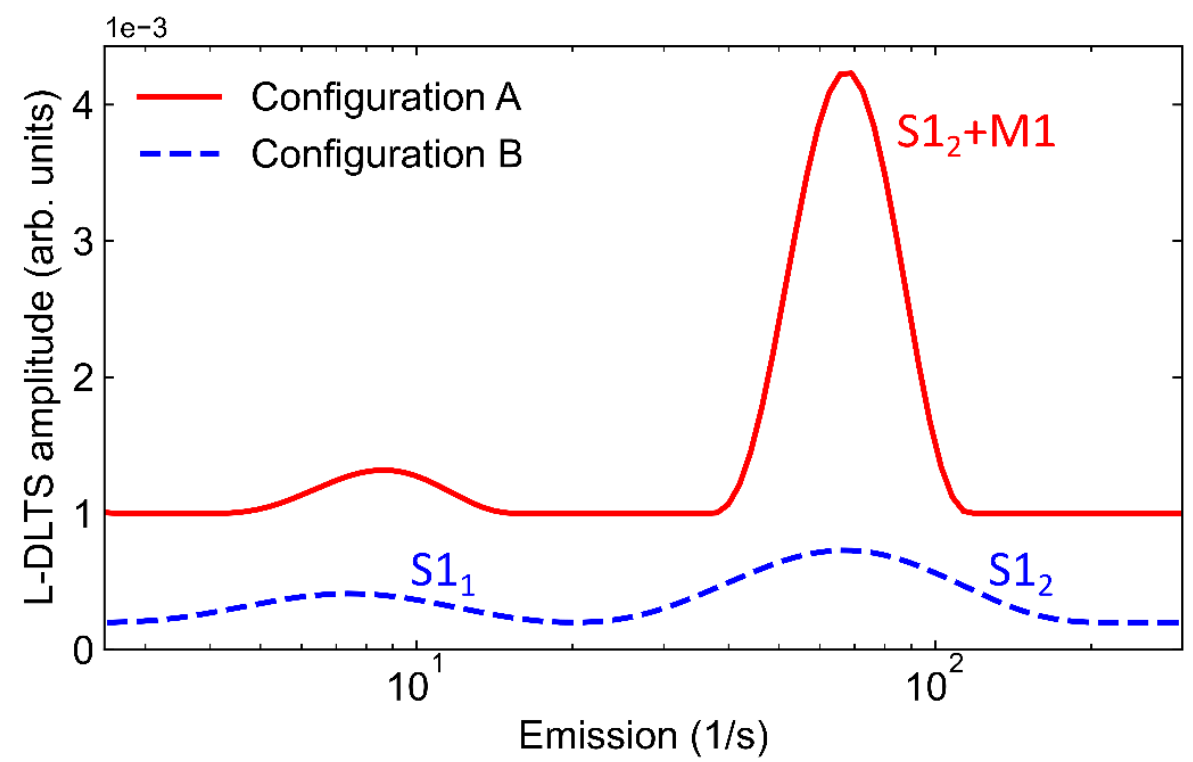

Figure 3. Laplace DLTS spectra for neutron irradiated sample in configurations A (red, solid) and B (blue, dashed), measured at $210 \mathrm{~K}$.

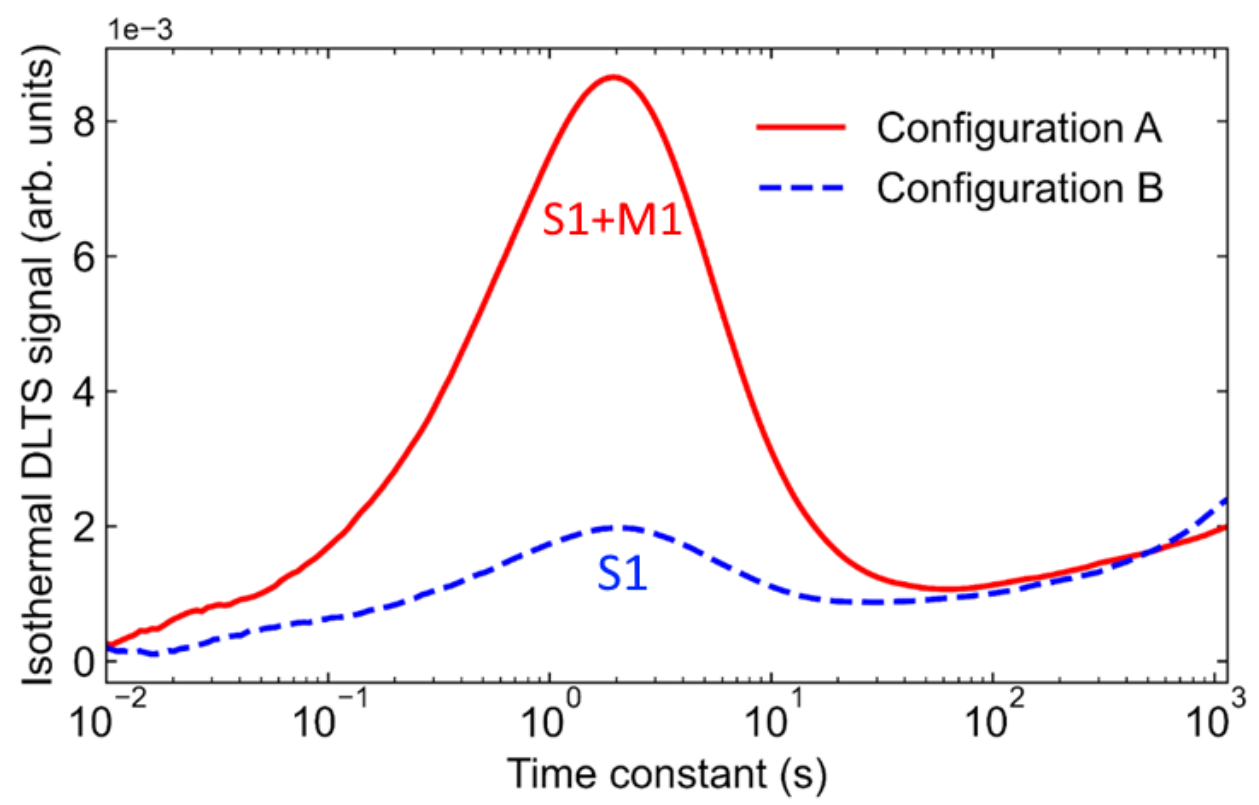

Figure 4. Isothermal DLTS spectra for neutron irradiated $4 \mathrm{H}-\mathrm{SiC}$ sample with $\mathrm{M}$-center in configurations A (red, solid) and B (blue, dashed). Measurements are done at $180 \mathrm{~K}$.

To compare the $\mathrm{M}_{1}$ deep level in $2 \mathrm{MeV}$ He ion implanted with epithermal and fast neutron irradiated $4 \mathrm{H}-\mathrm{SiC}$ in more detail, the isothermal DLTS has been applied. Figure 4 shows isothermal DLTS signals measured in configurations A and B. M-center was first placed in configuration B by annealing without bias at $450 \mathrm{~K}$ for $20 \mathrm{~min}$, which was followed by cooling down without bias and a $20 \mathrm{~min}$ long waiting period at a measurement temperature. Next, M-center was placed in configuration A by applying reverse bias (-30 $\mathrm{V}$ ) at the temperature of $340 \mathrm{~K}$ (for $20 \mathrm{~min}$ ) and cooling down under reverse bias $(-30 \mathrm{~V}$ ) to measurement temperature. The overlap of $\mathrm{S} 1$ and $\mathrm{M} 1$ is observed in configuration A, while the signal in configuration B is only due to S1 deep level. 
To get a "clean" signal coming only from the metastable defects, we have used the difference isothermal DLTS signal. The difference isothermal DLTS signal is obtained by subtracting isothermal DLTS spectra measured with M-center in configuration A and configuration B (Figure 6b). Such representation enables more accurate analysis, needed for deep level defect parameters estimation like activation energy, capture cross section, and concentration. More details on the analysis of isothermal DLTS data are given elsewhere [7]. Here, we provide an additional way of presenting isothermal DLTS signal using 2D plots.

Figure 5 shows heatmap plots of the difference in isothermal DLTS signal for (a) neutron irradiated (Figure 4) and (b) ion implanted samples (not shown here, see Ref. [7] for more details). As seen in Figure 5, the Mi signal is almost identical in both cases.

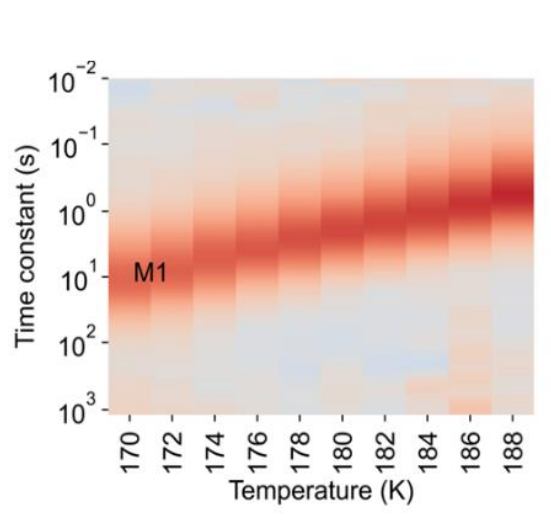

(a)

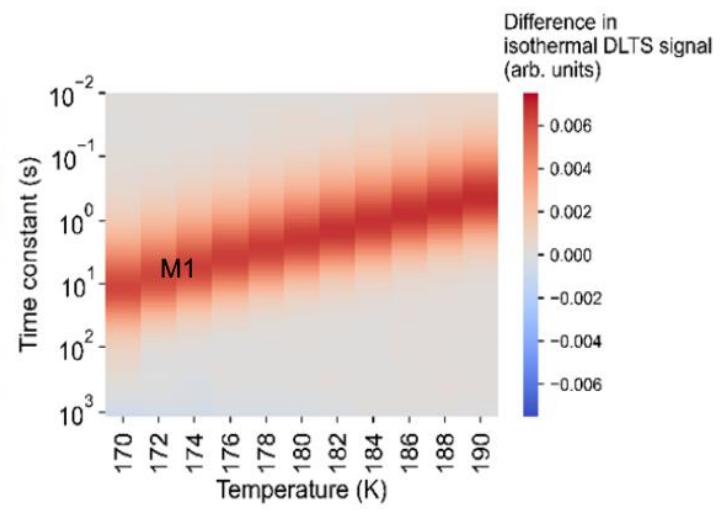

(b)

Figure 5. Heatmap plots of differential isothermal DLTS signal for (a) neutron irradiated and (b) ion implanted $4 \mathrm{H}-\mathrm{SiC}$ samples.

With $\mathrm{M}_{1}$ deep level being studied by L-DLTS and isothermal DLTS and results that clearly showed that $\mathrm{M}_{1}$ is indeed the same defect in both cases, neutron irradiated and ion implanted samples, we can move to a more complex situation, where the rest of $\mathrm{M}$ deep level defects $\left(\mathrm{M}_{2}, \mathrm{M}_{3}\right.$ and $\left.\mathrm{M}_{4}\right)$ overlap with $\mathrm{Z}_{1 / 2}, \mathrm{~S}_{2}$ and $\mathrm{EH}_{4}$. Unfortunately, due to the several closely spaced deep levels, L-DLTS cannot provide additional and accurate information. Therefore, we will focus on isothermal DLTS.

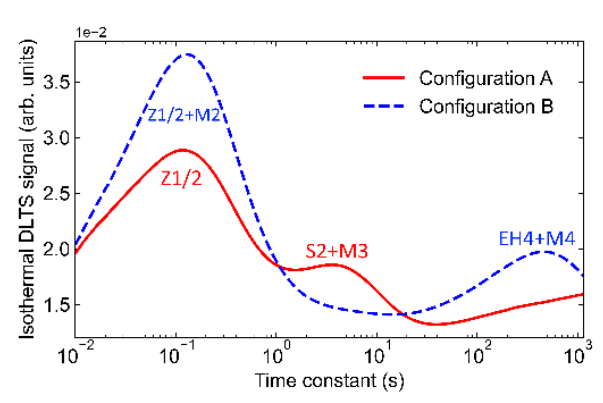

(a)

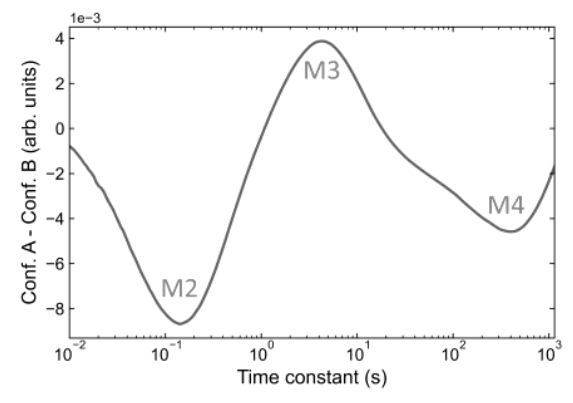

(b)

Figure 6. (a) Isothermal DLTS spectra of the M-center in configurations A (red, solid) and B (blue, dashed) measured at $300 \mathrm{~K}$, and (b) Difference isothermal DLTS signal (configuration A - configuration $\mathrm{B})$.

Figure 6a shows isothermal DLTS spectra of neutron irradiated sample with the Mcenter placed in configuration A and configuration B. The $\mathrm{M}_{2}$ and $\mathrm{M}_{4}$ deep levels observed 
in configuration $\mathrm{B}$ overlap with $\mathrm{Z}_{1 / 2}$ and $\mathrm{EH}_{4}$ deep levels, while the $\mathrm{M}_{3}$ deep level observed in configuration A overlaps with $S_{2}$ deep level. From the difference isothermal DLTS signal (Figure 6b), we can clearly detect signals due to the $\mathrm{M}_{2}, \mathrm{M}_{3}$ and $\mathrm{M}_{4}$ deep levels.

As seen in Figure 6b, the existence of the M4 deep level is once again confirmed using the isothermal DLTS. The obtained spectra match previously reported spectra in case of $2 \mathrm{MeV} \mathrm{He}$ ion implantation [7]. Using the all above mention techniques, we have estimated activation energies for metastable defects as follows: $\mathrm{M}_{1}(0.41-0.43 \mathrm{eV}), \mathrm{M}_{2}(0.64-$ $0.70 \mathrm{eV})$ and $\mathrm{M}_{3}(\sim 0.72 \mathrm{eV})$. The activation energy for $\mathrm{M}_{4}$ could not be accurately estimated due to the signal broadening. There are two possible explanations for this, i) the emission rate for $\mathrm{M}_{4}$ is too close to reconfiguration rate from configuration $\mathrm{B}$ to configuration $\mathrm{A}$, and ii) neutron irradiation has introduced more damage into material (compared to $2 \mathrm{MeV}$ He ion implantation), which has introduced more stress into the material, and increased the broadening of DLTS and isothermal DLTS signals [18].

Presented results, using the neutron irradiation, are consistent with all previously obtained and reported results on metastable defects in $4 \mathrm{H}-\mathrm{SiC}$ material using the low electron energy irradiation, proton irradiation and ion implantation [1-7]. Moreover, these results are consistent with the first-principal calculations of M-center [8]. Considering findings obtained in this study (such as bi-stability of M-center and the estimated energy levels) and by comparing it with the available literature data, we assign the M-center to the carbon self-interstitial.

\section{Conclusions}

We have investigated the $\mathrm{M}$-center introduced in $4 \mathrm{H}-\mathrm{SiC}$ by epithermal and fast neutron irradiation. Deep level defects arising from the metastable M-center were studied by means of junction transient techniques, DLTS, L-DLTS and isothermal DLTS. We have confirmed the existence of the fourth deep level defect, $\mathrm{M}_{4}$, already detected in ion implanted $4 \mathrm{H}-\mathrm{SiC}$ samples. Moreover, we have provided additional example of functionality of isothermal DLTS technique in studying the metastable deep level defects.

Author Contributions: Formal analysis, T.B.; investigation, T.B., I.C., T.M., V.R., L.S.; resources, T.M., V.R., L.S.; writing - original draft preparation, I.C..; writing-review and editing, T.B., I.C., V.R., L.S., T. M. All authors have read and agreed to the published version of the manuscript.

Funding: The present work was financially supported by the NATO Science for Peace and Security Programme, project no. G5674. The RBI project team would like to acknowledge financial support from the European Regional Development Fund for the Center of Excellence for Advanced Materials and Sensing Devices (Grant No. KK.01.1.1.01.0001).

Data Availability Statement: Data is contained within the article.

Acknowledgments: We would like to acknowledge Hidekazu Tsuchida and Norihiro Hoshino Central Research In-stitute of Electric Power Industry for the supply of SiC substrates with epitaxially grown $4 \mathrm{H}-\mathrm{SiC}$ single-crystal layers.

Conflicts of Interest: The authors declare no conflict of interest. 


\section{References}

1. Nielsen, H.K.; Martin, D.M.; Lévêque, P.; Hallén,A.; Svensson, B.G. Annealing study of a bistable defect in proton-implanted ntype 4H-SiC, Physica B: Condensed Matter 2003, 340-342, 743-747.

2. Beyer, F.C.; Hemmingsson, C.G.; Pedersen, H.; Henry, A.; Isoya, J.; Morishita, N.; Ohshima, T.; Janzén, E. Bistable defects in low-energy electron irradiated n-type 4H-SiC. Phys. Status Solidi Rapid Res. Lett. 2010, 4, 227-229.

3. Beyer, F.C.; Hemmingsson, C.; Pedersen, H.; Henry, A.; Janzén, E.; Isoya, J.; Morishita, N.; Ohshima, T. Annealing behavior of the EB-centers and M-center in low-energy electron irradiated n -type 4H-SiC. J. Appl. Phys. 2011, 109, 103703.

4. Martin, D.M.; Kortegaard Nielsen, H.; Lévêque, P.; Hallén, A.; Alfieri, G.; Svensson, B.G. Bistable defect in mega-electron-volt proton implanted $4 \mathrm{H}$ silicon carbide. Appl. Phys. Lett. 2004, 84, 1704-1706.

5. Nielsen, H.K.; Hallén, A.; Martin, D.M.; Svensson, B.G. M-center in low-dose proton implanted 4H-SiC; Bistability and change in emission rate. Mater. Sci. Forum 2005, 483-485, 497-500.

6. Nielsen, H.K.; Hallén, A.; Svensson, B.G. Capacitance transient study of the metastable M center in n -type $4 \mathrm{H}-\mathrm{SiC}$. Phys. Rev. B Condens. Matter Mater. Phys. 2005, 72, 085208.

7. Capan, I.; Brodar, T.; Bernat, R.; Pastuović, Ž.; Makino, T.; Ohshima, T.; Gouveia, J.D.; Coutinho, J. M-center in 4H-SiC: Isothermal DLTS and first principles modeling studies, J. Appl. Phys. 2021, 130, 125703.

8. Coutinho, J.; Gouveia, J.D.; Makino, T.; Ohshima, T.; Pastuović, Ž.; Bakrač, L.; Brodar, T.; Capan, I. M center in $4 \mathrm{H}-\mathrm{SiC}$ is a carbon self-interstitial. Phys. Rev. B 2021, 103, L180102.

9. Coutinho, J.; Torres, V.J.B.; Capan, I.; Brodar, T.; Ereš, Z.; Bernat, R.; Radulović, V. Silicon carbide diodes for neutron detection. Nucl. Inst. Methods Phys. Res. A 2020,

10. Brodar, T.; Bakrač, L.; Capan, I.; Ohshima, T.; Snoj, L.; Radulović, V.; Pastuović, Ž. Depth Profile Analysis of Deep Level Defects in 4H-SiC Introduced by Radiation. Crystals 2020, 10, 845.

11. Tokuda, Y. Transformation behavior of room-temperature-stable metastable defects in hydrogen-implanted n-type silicon studied by isothermal deep-level transient spectroscopy. J. Appl. Phys. 2006, 100, 023704.

12. Ziegler, J.F.; Ziegler, M.D.; Biersack, J.P. SRIM-The stopping and range of ions in matter (2010). Nucl. Instrum. Methods Phys. Res. Sect. B Beam Interact. Mater. Atoms. 2010, 268, 1818-1823.

13. Battistoni, G.; Cerutti, F.; Fassò, A.; Ferrari, A.; Muraro, S.; Ranft, J.; Roesler, S.; Sala, P.R. The FLUKA code: Description and benchmarking. AIP Conf. Proc. 2007, 896, 31-49.

14. Bathen, M.E.; Galeckas, A.; Müting, J.; Ayedh, H.M.; Grossner, U.; Coutinho, J.; Frodason, Y.K.; Vines, L. Electrical charge state identification and control for the silicon vacancy in 4H-SiC. NPJ Quantum Inf. 2019, 5, 111.

15. Son, N.T.; Trinh, X.T.; Løvlie, L.S.; Svensson, B.G.; Kawahara, K.; Suda, J.; Kimoto, T.; Umeda, T.; Isoya, J.; Makino, T.; et al. Negative-U System of Carbon Vacancy in 4H-SiC. Phys. Rev. Lett. 2012, 109, 187603.

16. Karsthof, R.; Bathen, M.E.; Galeckas, A.; Vines, L. Conversion pathways of primary defects by annealing in proton-irradiated n-type 4H-SiC, Phys. Rev. B 2020, 102, 184111.

17. Capan, I.; Brodar, T.; Coutinho, J.; Ohshima, T.; Markevich, V.P.; Peaker, A.R. Acceptor levels of the carbon vacancy in 4 H -SiC: Combining Laplace deep level transient spectroscopy with density functional modeling. J. Appl. Phys. 2018, $124,245701$.

18. Brodar, T.; Capan, I.; Radulović, V.; Snoj, L.; Pastuović, Z.; Coutinho, J.; Ohshima, T. Laplace DLTS study of deep defects created in neutron-irradiated n-type 4H-SiC. Nucl. Instrum. Methods Phys. Res. Sect. B Beam Interact. Mater. Atoms. 2018, 437, 27-31. 